\title{
A recurrent giant retroperitoneal myxoid liposarcoma: a case report and literature review
}

\author{
Shanshan Guo ${ }^{1,2 \#}$, Yingying $\mathrm{Xu}^{3 \#}$, Feng Qian ${ }^{4}$, Jingfan $\mathrm{Ma}^{5}$, Shaojun Wang ${ }^{3}$, Ping Chen ${ }^{1}$, Liang Zong ${ }^{1}$ \\ ${ }^{1}$ Department of Gastrointestinal Surgery, Northern Jiangsu People's Hospital, Clinical Medical College, Yangzhou University, Yangzhou 225001, \\ China; ${ }^{2}$ Department of Oncology, Graduate School of Medicine, Dalian Medical University, Dalian 116044, China; ${ }^{3}$ Department of General Surgery, \\ Yizhen People's Hospital, Yizhen 211400, China; ${ }^{4}$ Department of General Surgery, Southwest Hospital Affiliated to Army Medical University, \\ Chongqing 400038, China; ${ }^{5}$ Department of General Surgery, The First Hospital of Kunming, Kunming 650000, China \\ \#These authors contributed equally to this work. \\ Correspondence to: Liang Zong. Professor, Department of Surgery, Northern Jiangsu People's Hospital Affiliated to Yangzhou University, Yangzhou \\ 225001, China. Email: 250537471@qq.com; Feng Qian, MD. Department of General Surgery, Southwest Hospital Affiliated to Army Medical \\ University, No. 35, Gaotanyanzheng Street, Shapingba District, Chongqing 400038, China. Email: 506038746@qq.com.
}

\begin{abstract}
Retroperitoneal liposarcoma (RPLS) is a very rare type of tumor, accounting for less than $1 \%$ of all malignancies, especially the "large retroperitoneal liposarcoma" (GRPLS) of more than $20 \mathrm{~kg}$ (kilograms). Herein, we describe the treatment experience in a case of recurrent GRPLS. A 70-year-old woman was admitted with an enlarged abdomen, and computed tomography (CT) showed a large, lowdensity, homogeneous retroperitoneal mass $(40 \times 37 \times 26 \mathrm{~cm})$. In laparotomy, this $55 \times 40 \times 20 \mathrm{~cm}$ liposarcoma was completely removed and pathologically diagnosed as low-grade myxoid liposarcoma. The patient did not receive any adjuvant therapy, and CT showed no evidence of recurrence during follow-up.
\end{abstract}

Keywords: Retroperitoneal liposarcoma; giant liposarcoma; myxoid liposarcoma; recurrence.

Submitted Jun 10, 2019. Accepted for publication Sep 12, 2019.

doi: $10.21037 /$ tcr.2019.10.20

View this article at: http://dx.doi.org/10.21037/tcr.2019.10.20

\section{Introduction}

Liposarcoma is one of the most common primary retroperitoneal malignancies, accounting for approximately $10 \%$ of all soft tissue sarcomas. Due to the lack of significant anatomical space in the retroperitoneal cavity, these tumors typically grow to a large size and involve adjacent structures. Pathologically, retroperitoneal liposarcoma (RPLS) is currently classified into four types based on morphological features and cytogenetic aberrations, which are welldifferentiated, dedifferentiated, pleomorphic, and mucinlike/round cells. Low-grade liposarcoma (well-differentiated and myxoid) has a high local recurrence rate but a low metastatic rate. Modern imaging techniques are critical for diagnosis, type, stage, and follow-up. At present, the most important treatment for liposarcoma is still complete resection, and the effect of adjuvant therapy is controversial.

\section{Case presentation}

A 70-year-old woman was admitted with a complaint of abdominal enlargement after nine months of RPLS resection. She complained of nausea, vomiting, and a weight gain of $5 \mathrm{~kg}$. In the past three years, she had undergone four RPLS resections (September 2014, May 2015, October 2016, December 2016). She did not receive any adjuvant radiotherapy or chemotherapy after each operation. The physical examination revealed a large, soft, amorphous mass that extended down from the xiphoid to the pelvis, with a circumference of $93 \mathrm{~cm}$ and severe edema in the lower extremities. Laboratory findings and tumor markers were within the normal range. Computed tomography revealed a large amount of homogeneous retroperitoneal fat density extending down the subhepatic region to the pelvic cavity, moving the intraperitoneal structure to the left side of the 

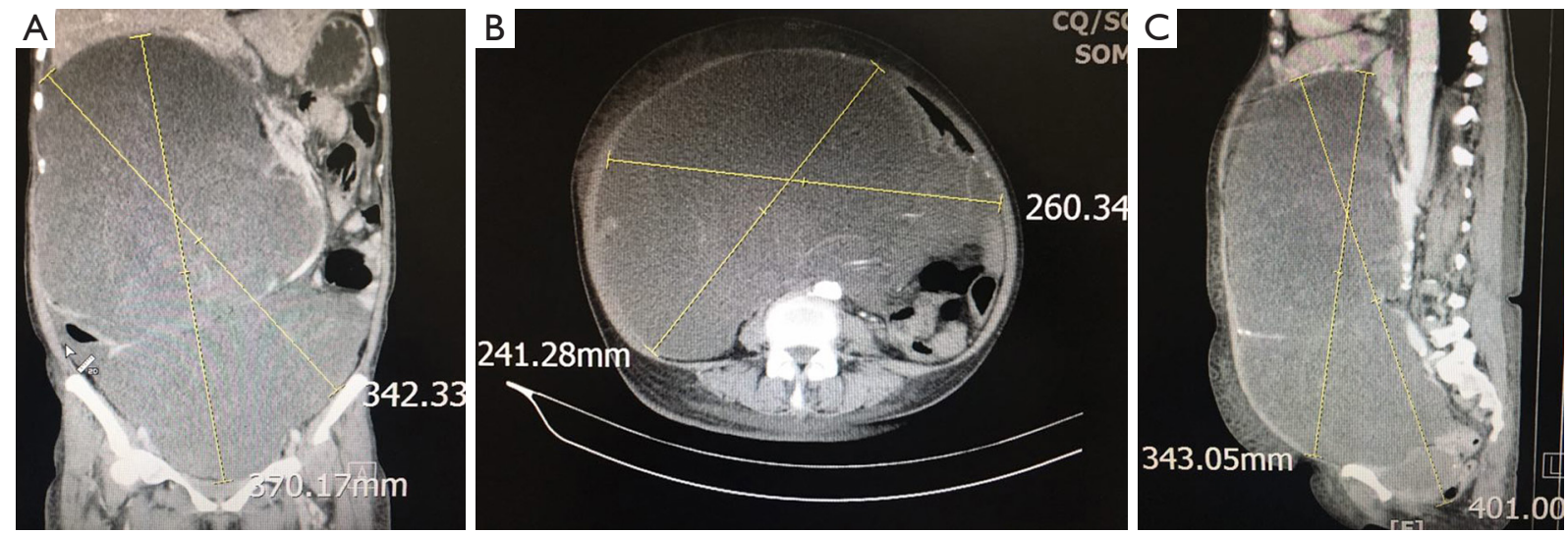

Figure 1 Computed tomography scans of the abdomen. A large homogeneous retroperitoneal mass displacing retroperitoneal structures dorsally with the largest diameter being $40 \times 37 \times 26 \mathrm{~cm}$ with the internal organs being pushed aside.
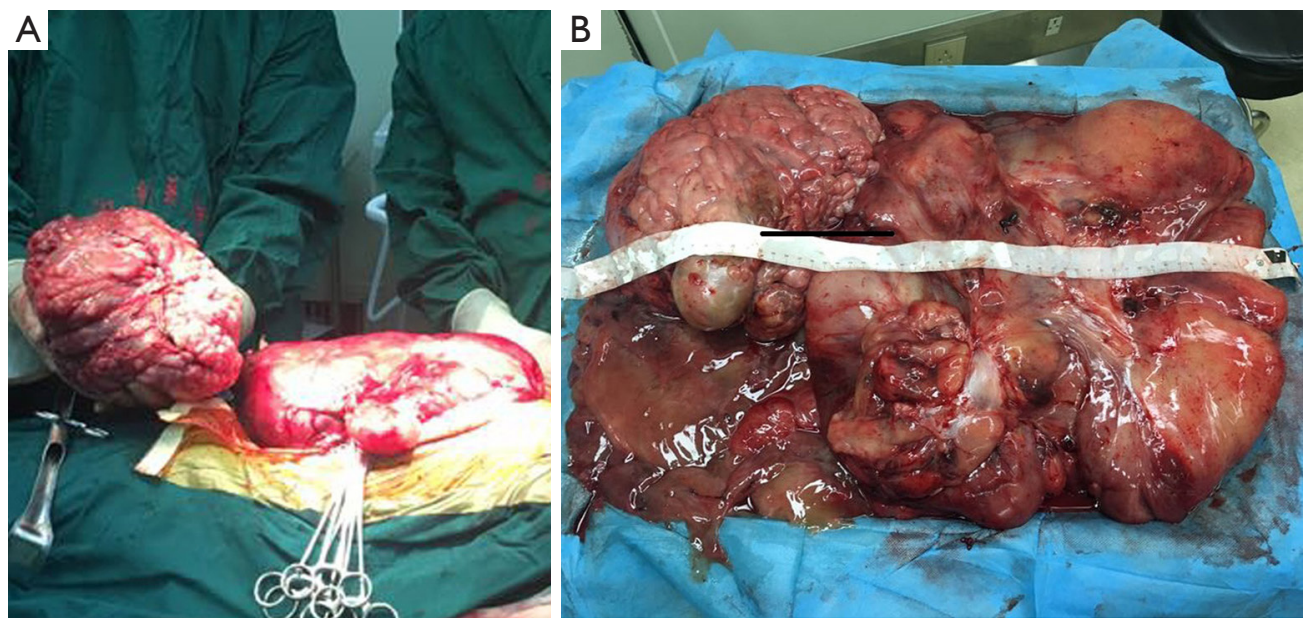

Figure 2 Intraoperative picture of the mass after laparotomy. The tumor was capsulated; the excised tumor was $55 \times 40 \times 20 \mathrm{~cm}$ and weighed $25 \mathrm{~kg}$. The black bar in the section (B) is $10 \mathrm{~cm}$.

abdomen (Figure 1).

In laparotomy, a giant amorphous and jelly mass was found that filled the abdominal cavity. After surgical exploration, the huge mass could be separated from two parts (abdominal part and pelvic part). We resected both masses completely. Meanwhile, another smaller mass was found and resected; all the above masses arose from the retroperitoneal space. Tumors measuring $55 \times 40 \times 20 \mathrm{~cm}$ were removed and weighed $28 \mathrm{~kg}$ (Figure 2).

The postoperative pathological report indicated myxoid liposarcoma. Immunohistochemical staining showed that this tumor was negative for STAT60, CD99, MDM2, SMA, and desmin, but positive for CDK4, CD34, Bcl-2, and $\mathrm{S}-100$. The patient was treated without any adjuvant therapy, experienced no postoperative complications, and was discharged 1 week after surgery. The patient presented no evidence of recurrence during follow-up by CT.

\section{Discussion}

Liposarcoma is the most frequent type of adult sarcoma, accounting for approximately $15 \%$ of soft-tissue sarcomas. Liposarcoma originates from multipotential mesenchymal cells, which are usually present in the lower extremities, and the retroperitoneum, inguinal, or paratesticular regions $(1,2)$.

Regarding the special localization, liposarcomas of retroperitoneal origin tend to form tumors of occasionally massive size. Retroperitoneal liposarcoma generally has 
Table 1 Clinical and histopathologic features, follow-up and clinical outcomes of ten patients with GRPLS in recent 10 years

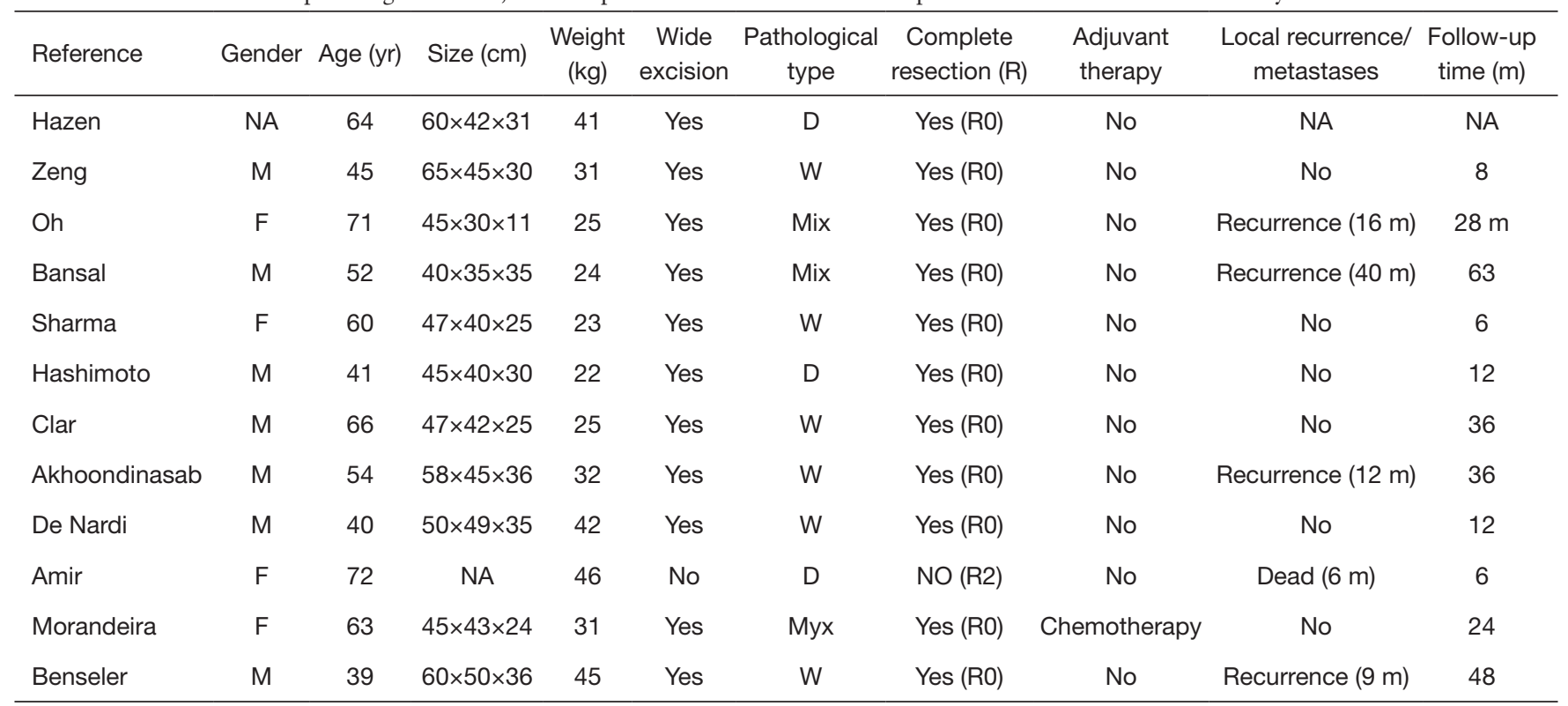

GRPLS, large retroperitoneal liposarcoma; F, female; M, male; D, dedifferentiated liposarcoma; W, well-differentiated liposarcoma; Mix, mix-type liposarcoma; Myx, myxoid liposarcoma; NA, not available; m, month.

an early silent growth period until the mass develops sufficiently to press against the circumferential architecture and often the surrounding structures, as a result of the flexibility of the peritoneum. Abodominal enlargement may be a unique symptom (3). Modern imaging techniques (CT, MRI) are essential during the preoperative stage for a complete and detailed study of the neoplasm, and are also critical during follow-up (4). CT or MRI scanning can show the shape, internal components, margins, and surrounding tissues. Different subtypes of retroperitoneal liposarcoma exhibit varying imaging features, and CT or MRI is an ideal method for diagnosing retroperitoneal liposarcoma. It has been assumed that, based on the high soft-tissue resolution, CT or MRI can discover and discern different subtypes of this tumor $(2,3,5)$. The differential diagnosis of retroperitoneal liposarcomas includes tumors with fatty components, such as renal angioleiomyolipoma, adrenal myelolipoma retroperitoneal lipoma, and teratoma (6).

For pathology, retroperitoneal liposarcoma is currently classified into four types: well-differentiated, dedifferentiated, myxoid/round cell, and pleomorphic (7). However, a minority of cases (5\% to $10 \%$ ) show a combination of two or three components (so-called mixed-type liposarcoma). Well-differentiated liposarcoma is the most common histological subtype, followed by myxoid liposarcoma (8). Low-grade liposarcoma (well- differentiated and myxoid) is unique and has a high rate of local recurrence but a low rate of distant recurrence. It is typically locally aggressive and has a tendency to recur after surgical excision even if no tumors have been initially resected with free margins. Low-grade liposarcoma also does not metastasize and very rarely dedifferentiates $(9,10)$. In our case, considering the high rate of local recurrence, we advised the patient to take adjuvant radiotherapy or chemotherapy; however, the patient refused. It has been increasingly important to regularly complete the follow-up.

The principle treatment for liposarcoma is complete resection. It is generally agreed that the most important factor influencing survival outcome of RPLS is the surgeon's skill in resecting the tumor completely (11). In our reviewed cases, just one case did not have complete resection (R2) and was the only death that occurred during the follow-up time. In addition, tumor histology, negative margins after surgery, metastases, and tumor size are independently associated with disease-specific survival, patients with decreased local recurrence rate after adjuvant therapies (12), and patients with local recurrence having lower survival rates (9).

We were also first to review the cases reported in the literature published in PubMed regarding giant RPLS (weight over $20 \mathrm{~kg}$ ) in the last 10 years. The clinical data are summarized in Table 1 (13-24). We found that only there are only 12 cases reported. There were 7 males (58.3\%), 
4 females $(33.3 \%)$ and one case had no available gender data, with a median age of 55.6 years (39-72 years). Among these 12 cases with a median weight of 32.25 years (22-46 years) kilograms, 7 cases $(58.3 \%$ ) are heavier than ours. In our reviewed cases, there are 6 cases $(50 \%)$ of welldifferentiated liposarcoma, 3 cases of $(25 \%)$ dedifferentiated liposarcoma, 2 (16.7\%) cases of mix-type liposarcoma, and 1 case of myxoid liposarcoma. The unique myxoid liposarcoma is a 63-year-old female, who underwent adjuvant chemotherapy after complete surgical resection. We found that there was no evidence of recurrence or death after surgery fir 2 years. Furthermore, in the 12 cases, only Amir's case did not have complete resection (R2 resection), and this patient died after 6 months. We recommend that surgical resection still be the first choice in the liposarcoma therapy.

\section{Conclusions}

We here report a case of a recurrent giant retroperitoneal myxoid liposarcoma in a 70-year-old female who was admitted with a complaint of an emergent abdominal enlargement. Computed tomography showed a large retroperitoneal mass from the subhepatic area down to the pelvic cavity. After laparotomy, the masses measured $55 \times 40 \times 20 \mathrm{~cm}$ and weighed $28 \mathrm{~kg}$. Postoperative pathological report showed myxoid retroperitoneal liposarcoma. Considering myxoid liposarcoma has a high rate of local recurrence but a low rate of distant metastasis, postoperative radiotherapy may improve local control. However, there was no adjuvant therapy in our case, and this may induce a bad prognosis, although we have advised the patient to take adjuvant therapy. A long-term follow-up examination is absolutely necessary as well.

\section{Acknowledgments}

Funding: None.

\section{Footnote}

Conflicts of Interest: All authors have completed the ICMJE uniform disclosure form (available at http://dx.doi. org/10.21037/tcr.2019.10.20). The authors have no conflicts of interest to declare.

Ethical Statement: The authors are accountable for all aspects of the work in ensuring that questions related to the accuracy or integrity of any part of the work are appropriately investigated and resolved. All procedures performed in studies involving human participants were in accordance with the ethical standards of the institutional and/or national research committee(s) and with the Helsinki Declaration (as revised in 2013). Written informed consent was obtained from the patient for publication of this case report and any accompanying images.

Open Access Statement: This is an Open Access article distributed in accordance with the Creative Commons Attribution-NonCommercial-NoDerivs 4.0 International License (CC BY-NC-ND 4.0), which permits the noncommercial replication and distribution of the article with the strict proviso that no changes or edits are made and the original work is properly cited (including links to both the formal publication through the relevant DOI and the license). See: https://creativecommons.org/licenses/by-nc-nd/4.0/.

\section{References}

1. Fabre-Guillevin E, Coindre JM, Somerhausen Nde S, et al. Retroperitoneal liposarcomas: follow-up analysis of dedifferentiation after clinicopathologic reexamination of 86 liposarcomas and malignant fibrous histiocytomas. Cancer 2006;106:2725-33.

2. Arvinius C, Torrecilla E, Beano-Collado J, et al. A clinical review of 11 cases of large-sized welldifferentiated liposarcomas. Eur J Orthop Surg Traumatol 2017;27:837-41.

3. Song T, Shen J, Liang BL, et al. Retroperitoneal liposarcoma: MR characteristics and pathological correlative analysis. Abdominal Imaging 2007;32:668-74.

4. Coli P, Sciandra PC, Marzano T. Myxoid liposarcoma of the retroperitoneum. Presentation of a clinical case. Minerva Chir 2000;55:537-40.

5. Lu J, Qin Q, Zhan LL, et al. Computed tomography manifestations of histologic subtypes of retroperitoneal liposarcoma. Asian Pac J Cancer Prev 2014;15:6041-6.

6. Salemis NS, Tsiambas E, Karameris A, et al. Giant retroperitoneal liposarcoma with mixed histological pattern: a rare presentation and literature review. J Gastrointest Cancer 2009;40:138-41.

7. Singer S, Antonescu CR, Riedel E, et al. Histologic subtype and margin of resection predict pattern of recurrence and survival for retroperitoneal liposarcoma. Ann Surg 2003;238:358-70; discussion 370-1.

8. Neuhaus SJ, Barry P, Clark MA, et al. Surgical 
management of primary and recurrent retroperitoneal liposarcoma. Br J Surg 2005;92:246-52.

9. Knebel C, Lenze U, Pohlig F, et al. Prognostic factors and outcome of Liposarcoma patients: a retrospective evaluation over 15 years. BMC Cancer 2017;17:410.

10. Tan MC, Brennan MF, Kuk D, et al. Histology-based Classification Predicts Pattern of Recurrence and Improves Risk Stratification in Primary Retroperitoneal Sarcoma. Ann Surg 2016;263:593-600.

11. Doglietto GB, Tortorelli AP, Papa V, et al. Giant retroperitoneal sarcomas: a single institution experience. World J Surg 2007;31:1047-54.

12. Lee HS, Yu JI, Lim DH, et al. Retroperitoneal liposarcoma: the role of adjuvant radiation therapy and the prognostic factors. Radiat Oncol J 2016;34:216-22.

13. Hazen B, Cocieru A. Giant Retroperitoneal Sarcoma. J Gastrointest Surg 2017;21:602-3.

14. Zeng X, Liu W, Wu X, et al. Clinicopathological characteristics and experience in the treatment of giant retroperitoneal liposarcoma: A case report and review of the literature. Cancer Biol Ther 2017;18:660-5.

15. Oh SD, Oh SJ, Suh BJ, et al. A Giant Retroperitoneal Liposarcoma Encasing the Entire Left Kidney and Adherent to Adjacent Structures: A Case Report. Case Rep Oncol 2016;9:368-72.

16. Bansal VK, Misra MC, Sharma A, et al. Giant

Cite this article as: Guo S, Xu Y, Qian F, Ma J, Wang S, Chen P, Zong L. A recurrent giant retroperitoneal myxoid liposarcoma: a case report and literature review. Transl Cancer Res 2019;8(7):2672-2676. doi: 10.21037/tcr.2019.10.20 retroperitoneal liposarcoma- renal salvage by autotransplantation. Indian J Surg 2013;75:159-61.

17. Sharma M, Mannan R, Bhasin TS, et al. Giant inflammatory variant of well differentiated liposarcoma: a case report of a rare entity. J Clin Diagn Res 2013;7:1720-1.

18. Hashimoto Y, Hatakeyama S, Tachiwada T, et al. Surgical treatment of a giant liposarcoma in a Japanese man. Adv Urol 2010;2010:943073.

19. Clar H, Leithner A, Gruber G, et al. Interdisciplinary resection of a giant retroperitoneal liposarcoma of $25 \mathrm{~kg}$. ANZ J Surg 2009;79:957.

20. Akhoondinasab MR, Omranifard M. Huge retroperitoneal liposarcoma. J Res Med Sci 2011;16:565-7.

21. De Nardi P, Bissolati M, Cristallo M, et al. Recurrent giant liposarcoma of the spermatic cord. Urology 2012;79:113-4.

22. Amir M, Akhtar S, Pervaiz M, et al. Giant dedifferentiated retroperitoneal liposarcoma. J Coll Physicians Surg Pak 2011;21:569-71.

23. Morandeira A, Prieto J, Poves I, et al. Giant retroperitoneal sarcoma. Can J Surg 2008;51:E79-80.

24. Benseler V, Obed A, Schubert T, et al. Case report--surgical therapy of a retroperitoneal liposarcoma weighing $45 \mathrm{~kg}$. Zentralbl Chir 2009;134:174-7. 